\title{
A atuação dos enfermeiros nos Grupos Operativos Terapêuticos na Estratégia Saúde da Família\#
}

The role of nurses in Operating Therapeutic Groups in the Family Health Strategy

\section{Resumo}

A pesquisa teve como objetivo conhecer a atuação dos Enfermeiros nos Grupos Operativos Terapêuticos na Estratégia Saúde da Família de um município da região sul de Santa Catarina. Trata-se de uma pesquisa de abordagem qualitativa, do tipo descritivo-exploratória e de campo. Utilizou-se como instrumento de coleta de dados a entrevista semiestruturada aplicada para 10 enfermeiros que atuam na Estratégia Saúde da Família. Utilizou-se para análise dos resultados as categorias de análise preconizadas por Minayo. Os resultados da pesquisa denotam a necessidade de sensibilização dos profissionais em relação aos Grupos Operativos Terapêuticos, instrumentalizando-os para desmistificar a visão curativista, além de promover nas atividades grupais o empoderamento dos sujeitos para a busca de uma melhor qualidade de vida.

Palavras-chave: Programa Saúde da Família. Educação em Saúde. Promoção da Saúde.

\begin{abstract}
The research aimed to meet the nurses ' performance in Operating Therapeutic Groups in the family health strategy of a municipality in the southern region of Santa Catarina. It is a qualitative research, exploratory and descriptive-type of field. Used as data collection instrument the semi-structured interview applied to ten nurses working in family health Strategies of that municipality. It was used for analysis of the results analysis categories proposed by Minayo. The search results denote the need to outreach to professionals in relation to Therapeutic Operating Groups, by actually Instrumenting them to demystify the vision curativista, in addition to promoting group activities empowerment of subject to the search for a better quality of life.
\end{abstract}

Keywords: Family Health Strategy. Health Education. Health Promotion.

DOI: $10.15343 / 0104-7809.20143804462472$

\# Artigo derivado da Monografia de Pós-Graduação: "Mazzuchello FR, Soratto MTS. A Organização dos Grupos Operativos Terapêuticos pelos Enfermeiros que atuam na Estratégia Saúde da Família. Criciúma (SC): Universidade do Extremo Sul Catarinense; 2012".

* Universidade do Extremo Sul Catarinense, Criciúma-SC, Brasil. Email: francielle_rosso@hotmail.com

** Universidade do Extremo Sul Catarinense, Criciúma-SC, Brasil. Email: luk@unesc.net

*** Universidade do Extremo Sul Catarinense, Criciúma-SC, Brasil. Email: mts@unesc.net

**** Universidade do Extremo Sul Catarinense, Criciúma-SC, Brasil. E-mail: vsd@unesc.net

***** Universidade do Extremo Sul Catarinense, Criciúma-SC, Brasil. E-mail: guiga@engeplus.com.br

Os autores declaram não haver conflitos de interesse. 


\section{INTRODUÇÃO}

A Educação em Saúde é um dos meios que interligam os profissionais da saúde e a população, estreitando os laços de aproximação. Ela é baseada em alguns princípios, como a escuta do outro; a troca de experiências e a construção de novos saberes, reconhecendo o outro como possuidor de um histórico de vida, trazendo consigo saberes, experiências e conhecimentos ${ }^{1}$.

Entende-se como grupo terapêutico um conjunto de indivíduos que possuem problemas semelhantes e reúnem-se para trocar experiências e conhecimentos e adquirir novas habilidades de superação, na busca por melhor qualidade de vida. A modalidade grupal como uma das práticas de educação em saúde possibilita aos participantes um empoderamento de conhecimentos, discussões, críticas e construção de práticas saudáveis referentes às questões de saúde, pois é no grupo que os participantes podem enfrentar de maneira mais adequada as suas limitações e resgatar também a sua autonomia para viver de um modo mais harmônico com as suas condições de saúde².

Quando os indivíduos participam de Grupos Terapêuticos é esperado que estes ampliem o controle sobre sua vida e modifiquem os contextos político e social nos quais estão inseridos, refletindo também na escolha do que é melhor para si, em consonância com suas crenças, valores, necessidades e realidade ${ }^{3}$.

Os Grupos Operativos Terapêuticos surgem como práticas educativas utilizadas pelo enfermeiro da Estratégia Saúde da Família (ESF), que, quando bem elaborados e planejados, possibilitam aprendizado e trocas de experiências, proporcionando interação, construção e aprimoramento da qualidade de vida dos sujeitos.

O profissional de saúde tem papel fundamental na educação em saúde, à medida que realiza vários tipos de ação, tais como: promover o desenvolvimento de conhecimentos e habilidades que contribuam para a adoção de estilos de vida mais saudáveis; estimular a participação efetiva da comunidade na construção da cidadania, na transformação de seu ambiente, na conquista da equidade social e em saúde, de forma que as pessoas possam modificar ativamente o ambiente e melhorar a qualidade de vida ${ }^{4}$.
O enfermeiro, como gestor da ESF, tem o objetivo de trazer a comunidade para mais perto de si, conhecendo a realidade do grupo populacional ao qual gerencia, promovendo o empoderamento dos indivíduos para o autocuidado, na perspectiva da educação em saúde como prática transformadora, sendo importante que os assuntos sejam discutidos de acordo com as necessidades do coletivo.

A proposta dos grupos terapêuticos pode possibilitar aos sujeitos mudança de comportamentos a partir da compreensão dos fatores relacionados ao processo saúde-doença, incorporando a vontade de mudar, transformar e apreender, na troca de saberes, tanto do sujeito como do profissional.

Para Pichon-Rivière, todo grupo tem um objetivo, uma tarefa que os sujeitos pretendem alcançar em conjunto. Esse objetivo pode ser o aprendizado de uma disciplina, de uma doença, a prestação de um serviço, ou mesmo um tratamento $^{5}$.

O planejamento das ações de saúde, muitas vezes, é realizado de maneira intuitiva ou pouco sistematizada. Quando se pretende alcançar objetivos complexos em conjunto, como acontece nos grupos terapêuticos, torna-se ainda mais evidente não só o quanto é importante planejar, como é, também, imprescindível contar com um método de planejamento ${ }^{6}$.

A educação em saúde não é apenas uma prática na qual o enfermeiro fala e a comunidade escuta; é uma permuta de conhecimentos com o outro e não para o outro. O exercício da prática de educação popular em saúde pressupõe abertura, disponibilidade para ouvir o outro, horizontalidade na relação interpessoal e na ação educativa em si, pois o ato participativo é humanizante ${ }^{7}$.

A mudança de perspectiva no pensar e fazer do enfermeiro no campo da educação popular em saúde agrega seu papel educativo ao resgate da pessoa como cidadã, participativa e consciente de sua condição de vida. No campo da saúde, em especial, da enfermagem, demanda um discurso e uma prática transformadores, mediado pela participação do sujeito em todo processo educativo ${ }^{7}$. 
Muitos dos profissionais que executam as atividades grupais desenvolvem-na de maneira empírica, baseadas no senso comum, por norma do programa, produtividade ou por "obrigação do serviço". O domínio do manejo do grupo deve ser pautado em um referencial teórico-prático que sustente essas atividades ${ }^{8}$.

As atividades de grupo devem fazer parte do cadores, a fim de modificar em conjunto, as atitudes que influenciam na qualidade de vida dos sujeitos. É um modo de diminuir a distância conceitual que se tem entre o conhecimento teórico do profissional enfermeiro e o obtido por meio das experiências da comunidade.

Dessa forma, o objetivo desta pesquisa foi conhecer a atuação dos Enfermeiros nos Grupos Operativos Terapêuticos na Estratégia Saúde da Família (ESF) de um município da região sul de Santa Catarina.

\section{MÉTODO}

Trata-se de uma pesquisa de abordagem qualitativa, descritiva e de campo. Foi realizada nas ESF, de um município do sul de Santa Catarina. Os sujeitos do estudo constituíram-se de 10 enfermeiros. A pesquisa teve como instrumento para coleta de dados a entrevista semiestruturada. Os dados foram analisados pela categorização de dados ${ }^{9}$.

A pesquisa foi aprovada pelo Comitê de Ética da Universidade do Extremo Sul Catarinense - UNESC, sob o protocolo n 176/2011.

Para preservar o sigilo decorrente da aplica-

ção das entrevistas, de acordo com as diretrizes e normas regulamentadoras que envolvem pesquisa com Seres Humanos, utilizou-se a letra "E" para os enfermeiros participantes da pesquisa, seguidos do respectivo número.

\section{RESULTADOS E DISCUSSÃO}

\section{Perfil dos Enfermeiros e Capacitação para Educação em Saúde}

Em relação ao perfil dos enfermeiros atuantes na ESF, a idade variou entre 29 e 51 anos; sexo feminino; com tempo de atuação entre 1 ano e 2 meses e 5 anos. Receberam capacitação

5 enfermeiros (E1, E2, E4, E5, E6) em curso introdutório para Saúde da Família (SF), o que incluía os grupos terapêuticos, além de Saúde Mental e Vigilância em Saúde. A capacitação foi promovida pela Secretaria Municipal de Saúde (SMS) e pela UNESC.

Pode-se destacar que 5 enfermeiros entrevistados (E3, E7, E8, E9, E10) referiram não receber capacitação sobre os Grupos Operativos Terapêuticos (GOT), sendo que o tempo de atuação variou de 1 ano e 4 meses a 4 anos.

A capacitação dos profissionais também contribui para melhores condições de trabalho, assim como aperfeiçoamento de relações entre os colaboradores com a comunidade. Há a necessidade de melhora em trabalhos com grupos e com o contato com o paciente que utiliza o serviço de Atenção Primária à Saúde (APS). Também é importante a integração da equipe e visão interdisciplinar das tarefas ${ }^{10}$.

Além de toda a capacidade técnica que os profissionais atuantes em ESF precisam ter, destacam-se a criatividade, força de vontade e perfil para se trabalhar em grupos. Deve haver uma mudança nos cursos de capacitação, para que sejam mais voltados para a integralidade e princípios da APS, assim como é necessária a utilização da aprendizagem significativa, por meio da Educação Permanente em Saúde ${ }^{11,12}$.

A atenção primária requer, cada vez mais, profissionais capacitados e que possuam perfil para tal. Também é necessária uma mudança nos cursos de capacitação para que sejam focados na integralidade e nos princípios da APS, e que sejam também mais baseados na aprendizagem significativa, por meio da Educação Permanente em Saúde ${ }^{11}$.

A atuação do enfermeiro como educador é de grande relevância, por possuir uma formação que o permite compreender as particularidades de cada sujeito e por assumir importante papel como componente essencial da equipe, pois além da função de coordenar, presta assistência como o cuidar, o gerenciar e o educar. O profissional da área de enfermagem, por meio de sua formação, concede ações prioritárias para redução de danos à saúde da população. Para tanto, aderir às práticas educativas na capacitação da equipe, por meio de educação continuada e da 
comunidade na educação permanente, torna-se fundamental para consolidar o propósito da $\mathrm{ESF}^{13}$.

\section{Grupos realizados na ESF}

Todos os enfermeiros presentes neste estudo realizavam atividades educativas nas escolas e creches vinculadas ao Programa Saúde na Escola (PSE) e Grupo de Hiperdia. Haviam também os Grupos de gestante, tabagismo, atividades educativas na comunidade e atenção domiciliar, educação continuada com a equipe, educação em saúde em temáticas especiais, conforme descrito no Quadro 1.

Quadro 1. Ações prioritárias realizadas pelos enfermeiros na ESF

\begin{tabular}{|l|l|}
\hline Educação em Saúde & Enfermeiros (E) \\
\hline $\begin{array}{l}\text { Programa Saúde na Escola (PSE); Grupos e Palestras nas } \\
\text { escolas e creches; Grupo Hiperdia }\end{array}$ & 10 (E1, E2, E3, E4, E5, E6, E7, E8, E9, E10) \\
\hline Grupos de gestantes & 07 (E1, E2, E3, E5, E8, E9, E10). \\
\hline Grupo de tabagismo & 04 (E2, E3, E7, E8) \\
\hline Grupo e Palestra com a comunidade & 02 (E3, E9) \\
\hline Educação Continuada com a equipe & 01 (E2) \\
\hline Orientação Domiciliar & 01 (E6) \\
\hline Saúde do Homem, Saúde da Mulher e Saúde da Criança & 01 (E5) \\
\hline Grupos épocas especiais & 01 (E2) \\
\hline
\end{tabular}

Fonte: Dados da pesquisa, 2012.

Todos os enfermeiros participavam do grupo de hiperdia mensalmente, com exceção da enfermeira E9, que, em função da pouca adesão dos hipertensos, participava dos grupos trimestralmente.

O Ministério da Saúde tem proposto uma série de medidas voltadas para o combate de doenças Crônicas, principalmente, para a Atenção Básica em Saúde. Assim, no ano de 2000, foi implantado o Plano de Reorganização da Atenção à hipertensão arterial e ao diabetes mellitus com o intuito de promover medidas preventivas e de promoção à saúde que assegurem uma melhor qualidade de vida a essas pessoas ${ }^{14}$.

Entre as medidas propostas para a prevenção, avaliação e o tratamento dessas morbidades, o Ministério da Saúde estabelece o desenvolvimento de atividades educativas individuais e em grupos para essa população. Os profissionais de saúde têm realizado as ações educativas, predominantemente, em grupos. Esses são conhecidos como Grupos Hiperdia, considerados espaços privilegiados de produção de cuidados $^{14}$.

Realizam grupos de gestantes 7 enfermeiros (E1, E2, E3, E5, E8, E9, E10).
O da Gestante está sendo readequado pela pouca adesão das mesmas, o planejamento está sendo feito para ser realizado uma vez por semana. (E1)

Grupo de gestantes uma vez por mês; Hiperdia, a medicação ocorre a entrega todo mês, mas o grupo não ocorre mensal porque os pacientes têm pouca adesão. Acabam ocorrendo de 3 em 3 meses. (E9)

Hiperdia mensalmente e gestante uma vez por semana. (E10)

Verifica-se nas falas que a periodicidade do grupo de gestante é semanal ou mensal, conforme a demanda e adequação das atividades nas unidades de saúde.

É primordial que os profissionais despertem para a gama de possibilidades de se trabalhar educação em saúde com gestantes que não somente as consultas individuais. Existem diversas estratégias que podem ser utilizadas pelos profissionais da ESF para a realização das ações básicas de saúde, preconizadas com o intuito de promover a saúde da população. Dentre essas estratégias, destacam-se as atividades de grupo que vêm sendo citadas em muitas pesquisas por 
se mostrarem uma ferramenta eficaz e adequada para uma assistência de qualidade ${ }^{15}$.

No grupo de tabagismo, 4 enfermeiros (E2, E3, E7, E8) realizavam atividades educativas, conforme descrito nas falas.

Hiperdia uma vez por mês; Gestante uma vez por mês e Tabagismo semanalmente. (E2)

Grupo de tabagismo mensalmente; grupo de gestante semanalmente e grupo hiperdia mensalmente. (E3)

Hiperdia mensal e o do Tabagismo conforme o protocolo do MS. (E7)

Hiperdia mensal; gestante semanal; tabagismo conforme o protocolo do MS; estamos no momento realizando um projeto para iniciar o grupo de crianças. (E8)

O grupo de tabagismo é realizado na periodicidade recomendada pelo Programa Nacional de Controle do Tabagismo do Ministério da Saúde.

No ano de 2000, durante o I Encontro de Consenso Nacional de Abordagem e Tratamento do Fumante, organizado pelo INCA e outras especialidades do setor, estabeleceu-se um documento universal a respeito das condutas empregadas no tratamento do fumante no Brasil. Esse documento considera algumas etapas para a cessação do fumo, como a abordagem cognitivo-comportamental, as terapias medicamentosas, os métodos alternativos e a abordagem para grupos especiais de pacientes ${ }^{16}$.

A enfermeira E5, além dos grupos de gestante e hiperdia, realizava um grupo específico para a "Saúde do Homem, Saúde da Mulher e Saúde da Criança anualmente" (E5). Já as enfermeiras E3 e E9 destacaram atividades educativas por meio de palestras, clube de mães e o arraial da saúde. A Enfermeria E2 destacou como ação prioritária a educação continuada com a equipe.

A enfermeira E6 realizava "orientações individuais e domiciliares, palestras nas creches e escolas". Ressalta-se na fala da enfermeira E6 as dificuldades inerentes ao trabalho com grupos em virtude da pouca adesão da população, atuação numa área considerada de risco com vulnerabilidade social e falta de estrutura física.
Só é realizado o hiperdia em grupos pequenos na sala de espera quando os mesmos vem buscar o medicamento, devido a pouca adesão da população nos grupos e por ser também uma população com risco acrescido (população de risco). Grupos mesmo não acontecem. (E6)

Para a viabilização dos Grupos Operativos Terapêuticos (GOT), é necessária a capacitação da equipe de enfermagem, estrutura física adequada e práticas pedagógicas que incentivem a adesão dos usuários.

Considerando as peculiaridades da ESF, comprova-se que a educação em saúde é uma das ações prioritárias incluídas no processo de trabalho das equipes. Esse modelo de atenção voltado às práticas educativas corresponde ao trabalho mais pertinente para o contexto da ESF, justificado pelo nível de compromisso e de responsabilidade esperado dos profissionais, pelo nível de adesão e participação da comunidade, pela ampliação do processo saúde-doença, pela humanização das práticas e pela busca incansável por uma assistência de qualidade ${ }^{17}$.

\section{Planejamento das ações de Educação em Saúde nos Grupos Operativos Terapêuticos}

A maioria dos enfermeiros (E1, E2, E4, E5, E7, E8, E9, E10) planejava as ações de EDS por meio de reuniões e discussões com a equipe, tendo o apoio do NASF (Núcleos de Apoio à Saúde da Família) (E1, E3, E4, E5, E7, E8, E9) e da Vigilância em Saúde (E1, E3), conforme destacado nas falas.

Geralmente só eu é quem faço os grupos. Pouca participação da equipe. Algumas vezes contamos com a presença do NASF e da Vigilância. Ocorrem mais em forma de palestra e conversação. (E3)

Os grupos são planejados nas reuniões de equipe. Os grupos de gestante e hipertenso já tem um cronograma de datas e assuntos que foi realizado no ano passado. Sempre ocorre a participação de toda a equipe e também do NASF. Os assuntos são abordados de forma dialogada e não palestra. (E8)

Os grupos são planejados nas reuniões de equipe, escolhemos os temas e o responsável 
por ministrar o grupo. Eles ocorrem em forma de palestra e com a participação da equipe de SF. (E10)

$\mathrm{O}$ atendimento do NASF deve sempre priorizar ações interdisciplinares e compartilhadas, com trocas de saberes e responsabilidades mútuas, gerando experiências para todos os profissionais envolvidos, utilizando metodologias como: estudos de caso, projetos terapêuticos, orientações e atendimento conjunto ${ }^{18}$.

É sabido, também, que a proposta inicial das ESF tem como especificidade a atuação de seus profissionais, que, além de competências técnicas, precisam ser criativos, com iniciativa e ter perfil para trabalhar com grupos. Entende-se, assim, que os profissionais necessitam de uma mudança em suas atuações para se adequarem a essas especificações, e que essas mudanças devem começar desde sua formação ${ }^{12}$.

A maioria dos enfermeiros realizava palestras expositivas dialogadas (E1, E2, E4, E7, E8, E10), com rodas de conversa (E1, E3, E9), além de dinâmicas de grupos (E4).

As ações são planejadas durante a reunião de equipe, a equipe discute para achar uma melhor forma de realizar os grupos, sempre pedimos apoio do NASF e da vigilância. Os grupos acontecem normalmente em forma de palestras, rodas de conversas. (E1)

Os grupos são realizados em forma de palestras e atividades dinâmicas, contamos sempre com a parceria do NASF e de toda a equipe (enf. Técnico de enf. ACS, Saúde Bucal). (E4)

Ocorrem mais em forma de conversa e palestra e com a participação da equipe e do NASF. Das gestantes todo mês escolho um tema para abordar e do hiperdia conforme a necessidade da população. (E9)

Considera-se essencial que a equipe multiprofissional da Estratégia Saúde da Família utilize meios estratégicos de Educação em Saúde que incentivem a participação da comunidade.

Além de ser da competência do educador realizar técnicas educativas apropriadas, é importante também a questão de reconhecer a cultura do indivíduo ou comunidade ${ }^{19}$.
Há, ainda, a predominância da dificuldade dos profissionais em interpretarem a realidade dos sujeitos, criando-se um distanciamento entre eles. É necessário que haja uma reflexão sobre a realidade e necessidade locais. O participante irá agir em conformidade com suas crenças e hábitos, por isso mesmo que a prática de EDS deve ser muito bem elaborada, pois se não for de acordo com a realidade deles não fará sentido algum e ficará perdida ${ }^{20}$.

Algumas competências se destacam ao enfermeiro educador. Entre elas citam-se: trabalhar de forma integral; entender a EDS como práxis, unindo teoria e prática; acolher e construir vínculos com a comunidade; reconhecer-se como agente educador e transformador; respeitar os modos de vida dos indivíduos; reconhecer que o saber profissional não é completo e dar valor ao saber popular; usar o diálogo realizando técnicas educativas que o enfatizem; dar informações adequadas e dar valor à intersetorialidade no cuidado à saúde ${ }^{21}$.

Destaca-se nas falas das enfermeiras E5 e E7 que para o planejamento das ações de EDS são consultados o Conselho Local de Saúde, a comunidade e a escola.

Planejamos os grupos com a equipe nas reuniões e participam também o conselho local de saúde, a comunidade e o NASF. (E5)

Todo fim de ano é realizado uma reunião com a equipe para planejar as ações de EDS para o ano todo. Contamos sempre com o apoio alem da equipe de SF do NASF, escola. Os grupos ocorrem em forma de palestras realizados normalmente pela enfermeira. (E7)

A aproximação dos profissionais de saúde com a comunidade faz com que cada vez mais sejam reconhecidos os limites do modelo médico assistencial e da Saúde Pública, assim como haja uma visão da realidade. Também contribui para que seja vencido o estigma de que os mais pobres e desfavorecidos precisam ser 'guiados' pelas elites de intelectuais e instâncias políticas ${ }^{22}$.

A enfermeira E2 realiza EDS voltada para a temática de épocas especiais, como o outubro rosa. 
Sempre ocorre a participação da equipe. Geralmente abordamos os assuntos da época, que estão em pauta, por exemplo, outubro fizemos o especialmente destinado as mulheres e também conforme a necessidade do público. Os grupos de hiperdia e gestante acontece mais em forma de palestras e orientações e o grupo de tabagismo é mais dinâmico, há mais interação, seguimos o protocolo do MS. (E2)

Destaca-se que a enfermeira E3 referiu a "pouca participação da equipe" no planejamento das ações educativas.

A enfermeira E6 não realiza grupos terapêuticos conforme preconiza o Ministério da Saúde. A realização dos grupos depende das especificidades da comunidade em que o enfermeiro atua de acordo com o perfil da população atendida. Além dessa questão, ressalta-se a necessidade de perfil da equipe multiprofissional da ESF para o processo de trabalho em EDS, o que demanda preparo na graduação, educação permanente da equipe, integração e participação efetiva.

A ESF surge como um novo modelo de cuidado da saúde tendo como foco a família e seu contexto social, tratando-a de forma integral e em todos os níveis de assistência, ampliando o processo saúde-doença, o vínculo e a continuidade do cuidado. Só é possível fortalecer esse processo se houver o trabalho em equipe e a integralidade da assistência prestada à população pela ESF²3 .

A integralidade do cuidado possibilita à equipe reconhecer os grupos mais vulneráveis, excluídos do sistema e com isso identificar as situações que requerem intervenções preventivas e educativas. Nessa perspectiva, a equipe estará organizando seu processo de trabalho direcionado aos princípios do SUS, levando a universalidade do cuidado e o acesso universal à totalidade dos usuários ${ }^{24}$.

\section{Avaliação das ações de Educação em Saúde nos Grupos Operativos Terapêuticos}

A avaliação das atividades educativas são realizadas, principalmente, pela observação do grupo, realizando perguntas e questionamentos sobre a temática e conversando com o grupo, de forma mais informal, segundo a maioria das enfermeiras (E1, E3, E5, E7, E8, E9).
Não tem um protocolo, existe apenas uma avaliação mais informal, observativa. (E3)

Sim. Tanto com a equipe como para a população sempre perguntamos no final se gostaram do grupo, do assunto. (E5)

Não tem um protocolo. A avaliação é mais informal. Na parte da escola os professores fazem a avaliação em forma de trabalho. (E7)

Para 3 enfermeiras (E1, E8, E9), a avaliação está relacionada à adesão e ao interesse da comunidade para participar dos grupos e ao próprio processo de aprendizagem.

Avaliamos observando a adesão da população e o interesse apresentado. (E1)

A avaliação ocorre mais na observação. Avaliamos conforme a adesão e o aprendizado da população. (E8)

Ocorre uma conversa, avaliamos o tema aplicado, a adesão, mas nada por escrito. (E9)

Segundo a enfermeira E2, o grupo de tabagismo é avaliado "através da quantidade de pacientes que deixaram de fumar" (E2).

Destaca-se na fala de E7 que a avaliação de Educação em Saúde realizada na escola, "é feita pelos professores em forma de trabalho".

A enfermeira E6 não realiza Grupos Operativos Terapêuticos de forma sistematizada e as enfermeiras E4 e E10 não realizam avaliação do processo educativo.

Considera-se essencial a avaliação das atividades educativas relacionadas aos grupos. Somente por meio da reflexão diária do processo de cuidar em enfermagem poderemos efetivar as práticas pedagógicas na EDS. Dessa forma, surgiram inquietações, tais como: A prática educativa é realizada de forma acessível para a comunidade? O enfermeiro está motivado para realizar as atividades de EDS? Utiliza práticas pedagógicas inovadoras e dinâmicas de grupos que propiciem a adesão dos usuários? Integra a equipe da ESF no processo de EDS? Como o enfermeiro realiza a autoavaliação no processo de trabalho em EDS?

É importante que o enfermeiro, enquanto coordenador e membro da equipe de saúde, esteja preparado para o enfrentamento e a resolução 
de problemas que correspondam, tanto em nível individual como coletivo, respeitando os princípios da ética / bioética, e propiciando condições para que os indivíduos vivam harmoniosamente, mesmo que haja limitações físicas, biológicas, sociais e ambientais na promoção à saúde ${ }^{13}$.

\section{Dificuldades para a realização dos Grupos Operativos Terapêuticos}

As enfermeiras E1, E2, E4, E7, E8, E10 consideravam como dificuldades a pouca adesão da comunidade aos grupos, sendo destacado nas falas de E1 e E2 que a população tem seu olhar voltado ao paradigma curativista, centrado no atendimento médico.

Pouca adesão no grupo de gestantes, elas priorizam mais a consulta médica. (E1)

Tenho dificuldades principalmente no grupo de hiperdia, pois eles não querem educação em saúde, prevenção, só querem a parte curativa, só querem a medicação. Exigem o direito de terem a medicação, mas não tem o comprometimento de participar dos grupos. (E2)

Uma das grandes dificuldades das equipes de saúde da família é trabalhar em um modelo pautado por práticas curativistas, com ênfase na doença e principalmente no atendimento médico ${ }^{18}$.

O modelo curativista ainda está fortemente interligado às práticas de saúde atuais, em que se valoriza o saber médico para promover hábitos e práticas de promoção da saúde e adesão da população aos procedimentos ${ }^{25}$.

A falta de participação da equipe foi mencionada como dificuldade para a realização dos grupos pelas enfermeiras E1 e E7.

A enfermeira E8 destacou os recursos humanos insuficientes para a população coberta como dificuldades para a organização dos grupos. A enfermeira E5 destacou a limitação de recursos materiais.

Em estudo realizado, observou-se que, entre muitas dificuldades, a que parecia ser mais significativa era a de equipes incompletas e sobrecarregadas de trabalhos. A maioria não contava com o profissional médico e com um número muito pequeno de ACS. Com a equipe incompleta, os profissionais optavam por não realizar atividades educativas diferenciadas ou mais elaboradas ${ }^{26}$.
Esse mesmo estudo cita que alguns suportes que precisam ser dados às equipes de ESF em relação às práticas de EDS são em relação a insumos e materiais pedagógicos, para que as ações de EDS sejam mais completas. Colocam, ainda, insumos para apoio técnico-pedagógico, para se autocapacitarem também em EDS e poderem realizar um trabalho mais qualificado ${ }^{26}$.

A falta de tempo e necessidade de dedicação do enfermeiro E7 foi ressaltada como dificuldade para a realização dos grupos.

Falta de interesse da população. Falta de tempo para dedicar aos grupos, o tempo é muito limitado na ESF, requer muita dedicação do enfermeiro em vários aspectos aí acabamos dividindo o nosso tempo e sobra pouco tempo para se dedicar aos grupos e também o desinteresse por parte de outros profissionais. (E7)

"A participação do próprio grupo no grupo" foi destacado como dificuldade pelo enfermeiro E3.

"A dificuldade de acesso da comunidade, caracterizada como área rural", foi citada como dificuldade para realizar o grupo pela enfermeira E9.

Os "temas a serem abordados" foram destacados como uma das dificuldades para a organização dos grupos terapêuticos pela enfermeira E1.

Vários são os empecilhos de caráter físico e estrutural que dificultam a realização de atividades educativas nas ESF. Entre eles estão: falta de salas adequadas, não disponibilidade de banheiros para a população, falta de ventilação, iluminação ruim, quantidade insuficiente de cadeiras e outros, que acabam por influenciar na qualidade das atividades prestadas ${ }^{26}$.

A enfermeira E6 novamente citou as características de risco da população atendida e as dificuldades em relação ao espaço físico.

Dificuldades por questão do espaço físico que a unidade é muito pequena, funciona num antigo bar e o local que pode ser feito que é um centro comunitário é muito longe e de difícil acesso da população, além de ser uma população de risco (usuários, profissionais do sexo), então eles não participam. (E6) 
Para a prática educativa se mostrar eficaz e satisfatória é importante que a equipe de saúde da ESF conheça a realidade da comunidade, dos indivíduos com os quais deseja-se realizar as ações educativas, suas potencialidades e suscetibilidades, adaptando essas ações educativas às necessidades, interesses e conhecimentos prévios de cada participante ${ }^{17}$. ao realizar a EDS, leve em consideração todo o contexto do paciente, que é também portador de uma história, tem seus conhecimentos e cultura. As situações em que a EDS ocorre são aquelas em que há a participação do sujeito em seu cuidado, transformando sua realidade e habilidades para lidar com a saúde ${ }^{27}$.

\section{Sugestão ao tema}

A capacitação da equipe com técnicas motivacionais e dinâmicas de grupos para a organização dos mesmos foi sugerido por 5 enfermeiras (E2, E3, E8, E9, E10).

Mais motivação para os profissionais estarem realizando os grupos, capacitações referentes aos temas abordados. (E2)

Orientações aos assuntos da atualidade para realizar os grupos, por exemplo: outubro rosa - saúde da mulher, vacinação do idoso, os temas que estão em foco. (E3)

Saber se organizar melhor, se preparar. Deveria existir educação continuada sobre técnicas para realizar os grupos. Dinâmicas nos grupos. (E8)

Capacitação referente aos grupos, ideias atuais, formas de realizar, algo que chame atenção do pessoal. (E9)

Apoio da SMS e capacitação referente aos grupos. (E10)

A necessidade da participação efetiva da equipe nos Grupos Operativos Terapêuticos e a diversificação de temas voltados à qualidade de vida foram destacadas nas falas de E1, E5 e E6.

Apoio da SMS, orientação e apoio de outros profissionais para abordar temas como atividade física, alimentação. (E1)

Buscar mais com a equipe em conjunto para encontrar os temas a serem abordados no grupo. (E5)

Ter um local apropriado para realizar os grupos e a participação do médico nos grupos. (E6)

O apoio da SMS (Secretaria Municipal de Saúde) foi citado pelas enfermeiras E1 e E10, e a necessidade de adequação da estrutura física foi sugerida pela enfermeira E6.

A falta de proximidade das Equipes de Saúde da Família e dos gestores com a comunidade faz com que não haja adesão às ações voltadas à EDS. Muitos profissionais colocam que a comunidade não adere às ações de EDS e tão pouco reconhece qual o papel de APS. Assim, a falta de elaboração de estratégias que levem em consideração o contexto sociocultural em que os sujeitos estão inseridos, os objetivos das equipes (prevenção e promoção) e os objetivos da população, de ter suas necessidades atendidas, nunca se adaptam ${ }^{12}$.

Tentar criar um protocolo para a realização de Educação em Saúde voltado para os grupos. (E4)

Será que a Educação em Saúde pode ser padronizada? As comunidades atendidas na ESF são substancialmente diferenciadas dependendo da área adstrita. Será que a comunidade deve se adequar a padrões educativos e preestabelecidos? Ou o enfermeiro é quem deve se adequar à realidade de cada comunidade inserida na ESF?

Entendidas como indispensáveis e complementares, saúde e educação fazem parte do processo de trabalho da ESF, assim como do cotidiano do enfermeiro. Existem inúmeras dificuldades nessa prática da EDS, porém alguns modos de diminuir esses obstáculos é o enfermeiro trabaIhar com a realidade de cada comunidade, criar vínculos com ela e identificar-se com os usuários. Assim, a operacionalização da EDS torna-se mais direcionada e eficaz à população e à equipe de saúde ${ }^{28}$.

Segundo a enfermeira E7, "dentro de cada SMS deveria existir uma equipe de profissionais que trabalhassem sobre EDS, grupos, para dar suporte para as unidades".

Ninguém melhor para promover EDS do que o enfermeiro que atua na ESF. Pressupõe-se que o 
enfermeiro é adequadamente capacitado para o trabalho em grupo e com os seres humanos desde a graduação. É ele quem conhece com propriedade a comunidade em que atua. É função dele a promoção da saúde.

A EDS é parte inerente das atribuições dos profissionais das equipes de USF e é ainda mais destacada no processo de trabalho da enfermagem. As próprias bases históricas e conceituais da enfermagem reconhecem o enfermeiro como educador. A EDS demanda do profissional uma análise crítica de sua atuação, bem como de seu papel como educador ${ }^{28}$.

O enfermeiro é um educador e deve ver-se como tal, já que os fundamentos teóricos da enfermagem preconizam a atribuição do enfermeiro como educador, afinal não há cuidar sem educar e vice-versa ${ }^{28}$.

Ressalta-se a importância das atividades grupais enquanto práticas educativas para o desenvolvimento humano, ajudando as pessoas a relacionar-se melhor consigo mesma, com o outro e com o mundo. As atividades grupais visam superar a visão educativa única e exclusivamente pautada na doença; procura empoderar o indivíduo a compreender melhor o processo saúde-doença, fazendo entender as condições de saúde que melhor lhe proporcionam benefícios, nas quais ele mesmo está apto a decidir sobre os determinantes e as condições da sua própria saúde a partir do entendimento que o grupo proporciona $^{29}$.

\section{CONCLUSÃO}

A Educação em Saúde é uma das formas que aproximam a comunidade da equipe de saúde. Permite a troca de saberes, criando experiências mútuas. Contribui para a criação de vínculo, estabelecendo relações de respeito e aprendizagem, além de permitir uma reflexão sobre as escolhas de cada indivíduo, melhorando sua qualidade de vida.

As atividades de grupo devem fazer parte do processo de trabalho do enfermeiro, como educadores, a fim de modificar, em conjunto, as atitudes que influenciam na qualidade de vida dos sujeitos. Para tanto, esses profissionais devem estar preparados e sensibilizados para as atividades grupais.

Em relação ao planejamento das ações realizadas nos grupos, foi constatado que a maioria dos enfermeiros planejou suas ações em conjunto com a equipe. A avaliação ocorreu em forma de observação, questionamentos e conforme o aprendizado dos sujeitos.

Por fim, como sugestão, os enfermeiros colocaram a necessidade de capacitação da equipe para a organização dos Grupos Operativos Terapêuticos como prioridade, além da participação efetiva da equipe nos grupos e a diversificação dos temas abordados, bem como, também, o apoio da equipe gestora.

O assunto é motivo de várias reflexões. Nesse âmbito, cabe salientar que o profissional enfermeiro participa diretamente das ações na atenção à saúde da família, sendo o responsável, inclusive, pelo planejamento e mediação dos Grupos Operativos Terapêuticos. Dessa forma, o enfermeiro e toda a sua equipe devem estar comprometidos com as ações e práticas de educação, promoção e prevenção à saúde planejada e executada para a comunidade, em prol de uma melhor qualidade de vida e o empoderamento crítico e construtivo dos sujeitos participantes dos grupos.

\section{REFERÊNCIAS}

1. David HMSL, Acioli S. Mudanças na formação e no trabalho de enfermagem: uma perspectiva da educação popular e de saúde. Rev Bras Enferm. 2010;63(1):127-31. DOI: http://dx.doi.org/10.1590/s0034-71672010000100021.

2. Soares LC, Santana MG, Thofehrn MB, Dias DG. Educação em saúde na modalidade grupal: relato de experiência. Ciên Cuid Saúde. 2009;8(1):118-23. DOI: http://dx.doi.org/10.4025/cienccuidsaude.v8i1.7786.

3. Besen CB, Netto MS, Da Ros MA, Silva FW, Silva CG, Pires MF. A estratégia saúde da família como objeto de educação em saúde. Saúde Soc. 2007;16(1):57-68. DOI: http://dx.doi.org/10.1590/s0104-12902007000100006.

4. Maciel ELN, Oliveira CB, Frechiani JM, Sales CMM, Brotto LDA, Araújo MD. Projeto Aprendendo Saúde na Escola: a experiência de repercussões positivas na qualidade de vida e determinantes da saúde de membros de uma comunidade escolar em Vitória, Espírito Santo. Ciên Saúde Colet. 2010;15(2):389-96. DOI: http://dx.doi.org/10.1590/s1413$\underline{81232010000200014 .}$ 
5. Castanho PCG. O momento da tarefa no grupo: aspectos psicanalíticos e psicossociais. Rev SPAGESP. 2007;8(2):13-22.

6. Campos FCC, Faria HP, Santos MA. Planejamento e avaliação das ações em saúde. 2a ed. Belo Horizonte: Nescon / UFMG / Coopmed; 2010. 114 p.

7. Alvim NAT, Ferreira MA. Perspectiva problematizadora da educação popular em saúde e a enfermagem. Texto Contexto Enf. 2007;16(2). DOI: http://dx.doi.org/10.1590/s0104-07072007000200015.

8. Lucchese R, Munari DB, Medeiros M. Reflexões sobre o uso de atividades grupais na atenção a portadores de doenças crônicas. Ciên Cuid Saúde. 2009;8:148-54. DOI: http://dx.doi.org/10.4025/cienccuidsaude.v8i0.9742.

9. Minayo MCS. Pesquisa social: teoria, método e criatividade. 28a ed. Petrópolis (RJ): Vozes; 2009. 108 p.

10. Cotta RMM, Schott M, Azeredo CM, Franceschini SCC, Priore SE, Dias G. Organização do trabalho e perfil dos profissionais do Programa Saúde da Família: um desafio na reestruturação da atenção básica em saúde. Epidemiol Serviços 472 Saúde. 2006;15(3). DOI: http://dx.doi.org/10.5123/s1679-49742006000300002.

11. Silva JAM, Ogata MN, Machado MLT. Capacitação dos trabalhadores de saúde na atenção básica: impactos e perspectivas. Rev Eletrônica Enf. 2009;9(2):389-401. DOI: http://dx.doi.org/10.5216/ree.v9i2.7173.

12. Ronzani TM, Silva CM. O Programa Saúde da Família segundo profissionais de saúde, gestores e usuários. Ciên Saúde Colet. 2008;13(1):23-34. DOI: http://dx.doi.org/10.1590/s1413-81232008000100007.

13. Amaral LR, Oliveira MAD, Cardoso RB, Ávila SPAR, Cardoso BLC. Atuação do enfermeiro como educador no programa saúde da família: importância para uma abordagem integral na atenção primária. FG Ciên Guanambi. 2011;1(1):1-21.

14. Melo LP, Canha BG, Ramos DC, Silva NP, Silva KCL, Bernardo VG, et al. A experiência de estudantes de Enfermagem em um grupo de educação em saúde: uma abordagem dialógica. Rev Bras Promoção Saúde. 2011;24(2):180-8.

15. Vasconcelos MCT, Machado MFAS, Becker SLM. Educação em Saúde a gestantes utilizando a estratégia grupo. Rev Rene. 2007;8(3):107-16.

16. Brasil. Ministério da Saúde. Instituto Nacional de Câncer José Alencar Gomes da Silva. Brasília: Ministério da Saúde; 2012.

17. Roecker S, Budó MLD, Marcon SS. Trabalho educativo do enfermeiro na Estratégia Saúde da Família: dificuldades e perspectivas de mudanças. Rev Esc Enferm USP. 2012;46(3):641-9. DOI: http://dx.doi.org/10.1590/s008062342012000300016.

18. Brasil. Ministério da Saúde. Diretrizes do NASF: Núcleo de Apoio a Saúde da Família. Brasília: Ministério da Saúde; 2010. $152 \mathrm{p}$.

19. Boehs AE, Monticelli M, Wosny AM, Heidemann IBS, Grisotti M. A interface necessária entre enfermagem, educação em saúde e o conceito de cultura. Texto Contexto Enf. 2007;16(2). DOI: http://dx.doi.org/10.1590/s0104-07072007000200014. 20. Figueiredo MFS, Rodrigues-Neto JF, Leite MTS. Modelos aplicados às atividades de educação em saúde. Rev Bras Enf. 2010;63(1). DOI: http://dx.doi.org/10.1590/s0034-71672010000100019.

21. Leonello VM, Oliveira MAC. Construindo o diálogo entre saberes para ressignificar a ação educativa em saúde. Acta Paulista Enf. 2009;22.

22. Vasconcelos EM. A educação popular na atenção primária à saúde. Rev Atenção Primária Saúde. 2008;11(3):344-5.

23. Kell MCG, Shimizu HE. Existe trabalho em equipe no Programa Saúde da Família? Ciên Saúde Colet. 2010;15(Supl 1):1533-41. DOI: http://dx.doi.org/10.1590/s1413-81232010000700065.

24. Fernandes LCL, Bertoldi AD, Barros AJD. Utilização dos serviços de saúde pela população coberta pela Estratégia de Saúde da Família. Rev Saúde Pública. 2009;43(4):595-603. DOI: http://dx.doi.org/10.1590/s0034-89102009005000040.

25. Freitas FV, Rezende Filho LA. Modelos de comunicação e uso de impressos na educação em saúde: uma pesquisa bibliográfica. Interface. 2011;15(36):243-56. DOI: http://dx.doi.org/10.1590/s1414-32832010005000044.

26. Sampaio J, Santos RC, Paixão LA, Torres TS. Promoção da saúde sexual: desafios no Vale do São Francisco. Psicol Sociedade. 2010;22(3). DOI: http://dx.doi.org/10.1590/s0102-71822010000300010.

27. Dias VP; Silveira DT, Witt RR. Educação em saúde: o trabalho de grupos em atenção primária. Rev Atenção Primária Saúde. 2009;12(2):221-7.

28. Fernandes MCP, Backes VMS. Educação em saúde: perspectivas de uma equipe da Estratégia Saúde da Família sob a óptica de Paulo Freire. Rev Bras Enferm. 2010;63(4):567-73. DOI: http://dx.doi.org/10.1590/s0034-71672010000400011.

29. Combinato DS, Vecchia MD, Lopes EG, Manoel RA, Marino HD, Oliveira ACS, et al. "Grupos de Conversa": saúde da pessoa idosa na estratégia saúde da família. Psicol Sociedade. 2010;22(3):558-68. DOI: http://dx.doi.org/10.1590/s010271822010000300016. 